\title{
Energy-neutral Wireless-powered Networks
}

\author{
Oktay Cetinkaya, Member, IEEE, Ergin Dinc, Member, IEEE, Caglar Koca, Student Member, IEEE, \\ Geoff V. Merrett, Senior Member, IEEE, and Ozgur B. Akan, Fellow, IEEE
}

\begin{abstract}
The Internet of Things (IoT) is a key enabler for remote monitoring and control of any medium with wireless devices deployed in substantial numbers. However, these devices often lack the desired lifetimes due to their incompetent batteries. If the envisaged scale of the IoT is realized, replenishing millions of batteries will become impractical. To address this issue, joint utilization of two prominent technologies, energy harvesting (EH) and wireless power transfer (WPT), is explored in this paper. By coupling data from empirical measurements on EH profiles with Federal Communications Commission (FCC) regulations on indoor WPT, we propose and numerically evaluate design guidelines for energy-neutral wireless-powered networks, in which a source first extracts energy from its medium and then uses the collected energy to operate wireless devices via WPT. The initial findings reveal that the IoT devices in a $100 \mathrm{~m}^{2}$ office building can be remotely energized by only three EH-enabled wireless power transmitting sources validating the proposed architecture.
\end{abstract}

Index Terms-Energy Harvesting, Wireless Power Transfer, Internet of Things, Energy-neutral, Wireless-powered Networks

\section{INTRODUCTION}

The unprecedented proliferation of the Internet of Things (IoT) has further enabled prevalent and ubiquitous adoption of wireless devices into diverse domains [1]. However, existing technology fails in keeping pace with the demands of ever-evolving IoT services. Here, the foremost drawback is limited-capacity batteries that these devices depend on. Due to randomness in depletion times, battery replenishment, i.e., maintenance, is no longer an option for widespread IoT applications [2]. Therefore, research has recently focused on solutions that may turn these energy-craving devices into selfsustaining entities, which are free from resource constraints.

To alleviate limited device lifetimes, two main approaches, namely energy harvesting $(\mathrm{EH})$ and wireless power transfer (WPT), come into prominence. EH is the process of transforming any ambient resource into a readily utilizable form [3], whereby an auxiliary reservoir is opportunistically generated. Besides, WPT is the conveyance of energy in radio frequency (RF) waves to power wireless devices in a remote manner [4]. Considering unique capabilities, utilization of both at the same time sounds highly profitable in overcoming ongoing issues.

This paper investigates the feasibility of an energy-neutral IoT network, components of which, from sensors to Access Points (APs), do not rely upon any external supply, such as mains electricity and/or batteries. Wireless devices in this

O. Cetinkaya and G. V. Merrett are with School of Electronics and Computer Science, University of Southampton, Southampton SO17 1BJ, UK e-mail: $\{$ oc1y18, gvm $\}$ @ecs.soton.ac.uk.

E. Dinc, C. Koca and O. B. Akan are with Internet of Everything (IoE) Group, Electrical Engineering Division, University of Cambridge, Cambridge CB3 0FA, UK. e-mail: \{ed502, ck542, oba21\}@cam.ac.uk.

This work was partially supported by the UK EPSRC under EP/P010164/1. Simulation data can be found at DOI 10.5258/SOTON/D0928. setting, i.e., sensor nodes, are operated via WPT by RF power sources harvesting energy from a domain-specific ambient resource. Specifically, electric field (E-field) EH [5], the highest energy-yielding indoor EH technique [6], is considered in this paper to validate the concept via realistic EH profiles gathered by empirical measurements [7]. For outdoor, any technique (e.g., solar EH) can be employed without loss of generality.

The main contributions besides proposing a novel network architecture are to tackle the discrepancies between the theory and practice: assuming an unlimited power source for WPT, and neglecting transmit power regulations. For the remote energization of wireless devices, a limited supply with intermittent availability, i.e., an EH source of power, is considered for the first time in literature. Based upon this agenda, we analyze a wireless-powered indoor IoT network complying the regulations on the maximum allowed transmit power enforced by the FCC. Design guidelines ensuring successful coverage with a minimum number of sources are accordingly evaluated. Simulation results obtained with the parameters derived using empirical data reveal that totally untethered and maintenancefree IoT networks can be practically implemented.

\section{System AND NeTwORK MODEL}

Here, we first introduce the employed EH technique acquiring the energy budget required to power wireless devices. This is followed by operation overview, and communication model explaining nodal cycles. Then we derive analytical expressions of the design guidelines assuring effective power coverage.

\section{A. Employed EH Technique and WPT Architecture}

The energy used on the wireless devices, i.e., the power transferred by the RF source, is extracted from the E-field in the vicinity of overhead fluorescent fixtures. This wireless $\mathrm{EH}$ technique simply enables the exploitation of E-field induced electric charges by means of displacement current [8]. The energy collected is then transferred to the devices via WPT using RF waves. Here, the EH and WPT processes are independent; hence, any EH technique supplying the sufficient power output required for WPT can be utilized without any reserve.

The empirical results disclosed in [7] show that it is possible to harvest $3.92 \mathrm{~J}$ of energy in $10 \mathrm{mins}$ with the implied $\mathrm{EH}$ technique. In short, the RF source periodically collects $\simeq 4 \mathrm{~J}$ of energy and transmits $2.5 \mathrm{~J}$ of it, since it needs $1.5 \mathrm{~J}$ of energy during EH and WPT, and for performing communicationand networking-related tasks, such as data collection and coordination, at an average power consumption of $2.5 \mathrm{~mW}$ [9].

Contrary to our previous efforts [10], [11] and the related existing works in literature, we inspect the applicability of powering wireless devices with a finite source of power that 
harvests energy from its medium. Our simulations revealing the design guidelines are based solely on the experimental data obtained by our prototypes running on real-life test beds [7].

\section{B. Operation Overview}

The system is designed in a hierarchical order employing "point-to-multipoint", i.e., star topology for WPT from RF sources to wireless-powered devices (w-p $D \mathrm{~s})$. The $\mathrm{w}-\mathrm{p} D$ operates like a sensor node without battery, which observes its environment and reports the parameters of interest back to the RF source that behaves as an AP after power transmission. Since the energy required for w-p $D$ 's operation is delivered by a remote source of power, namely RF source, the w-p $D$ s perform their tasks as long as they intercept enough power.

Expectedly, replenishing a w-p $D$ with more than one source through WPT provides higher energy for the same time period or delivers the required energy in a shorter duration. This certainly increases the reporting frequency of the w-p $D$ s as the time spent on EH for both sources and devices is shortened. However, here we assume that power transfer from sources to devices and the communication between them are performed in the same frequency band. Thus, overlapping coverages of the RF sources may cause interference, which degrades communication reliability due to losses and channel errors [11]. Therefore, to avoid interference, we proceed with one source for one device approach, in which a $\mathrm{w}-\mathrm{p} D$ is fed by only one RF source. It should be noted that this is the worstcase in terms of duty cycling, but one of the best setting for the number of sources required to cover the same volume.

Fig. 1 illustrates the planned application scenario in an office building, where RF sources remotely operate the w-p $D$ s located in their non-overlapping circular coverages of $\pi R_{R F}^{2}$.

\section{Communication Model and Nodal Operations}

As explained, the w-pDs exploit the RF source dissipated RF waves to sustain nodal operations, i.e., wake-up, sensing, processing, and communication. After being processed, the w$\mathrm{p} D$ data are conveyed back to the Internet-enabled RF source in a single hop. This setting refers to a basic IoT scenario, in which an upper-level authority is alerted about any parameter of interest for further decision-making procedures. In other words, apart from power provisioning, RF sources operate also as APs envisioning concentric coordinators that manage both power and data transitions throughout the network.

The following set of facts and assumptions are also considered for the operation of network components:

- The network relies on the energy harvested by the RF source, profile of which is determined by real-life experiments as explained in Section II-A. There is no other source of power feeding the network, where the existing one has the limited capacity with intermittent availability.

- The w-pDs are supplied by the RF source transmitting $P_{T}$ of power for a duration of $T_{T}$ in a periodic manner.

- The w-p $D$ s are accepted to be "asleep" before they perform their nodal operations, meaning that from when the RF source starts harvesting energy till it ends transmitting



(a)



(b)
Figure 1: (a) Orientation of network components (cross-sectional view); (b) Depiction of RF source coverage and w-p $D$ deployment in an event area, i.e., office building, of size $\Delta$ (aerial viewpoint).

power, they are in ultra-low-power mode. During this sleep state, the w-pDs consume $E_{S}$ of energy.

- The w-pDs follow a "harvest-then-operate" scheme, where they first extract energy from the RF waves transmitted by an RF source via WPT and then perform nodal operations, each of which requires a certain amount of energy resulting in a total of constant consumption $E_{N}$.

- The sum of $E_{S}+E_{N}$ gives the minimum required energy $E_{R}^{m i n}$, which needs to be collected by the w-p $D$ s in each cycle for effective execution of their designated tasks.

- Since the EH profile of RF sources changes minimally in time due to the stable operation of illumination assets, i.e., constant E-field presence, they are considered as generic current sources with adjustable output. Therefore, the w$\mathrm{p} D$ s are accepted to receive the same power in each cycle.

\section{Transmission Coverage}

The received power $P_{R}$ by a w-p $D$ at a distance $R_{R F}$ due to an RF source performing EM wave-based WPT [4] at transmit power $P_{T}$ can be given by Friis Transmission Formula [12] as

$$
P_{R}=P_{T} \frac{G_{T} G_{R} \lambda^{2}}{(4 \pi)^{2} R_{R F}^{\delta}}, \text { and } \lambda=\frac{c}{f},
$$

where $G_{T}$ and $G_{R}$ are respectively antenna gains of the RF source and the w-p $D, \lambda$ is wavelength, $c$ is the speed of light, $f$ is carrier frequency of the source, and $\delta$ is path loss exponent. In [13], $\delta$ is empirically measured as 2.04 when the device is in line-of-sight (LoS) of the source, and 2.94 for the non-LoS case, in an office building.

The generic WPT model given by (1) indicates the incident power on the $\mathrm{w}-\mathrm{p} D$, which needs to be converted into usable DC voltage by a rectifying antenna (rectenna). Therefore, the effective power $P_{R^{e f f}}$ that the w-p $D$ can utilize is usually calculated by the following linear model

$$
P_{R^{e f f}}=\eta P_{R}
$$

where $\eta$ is the RF-to-DC conversion efficiency. In practice, $\eta$ increases with $P_{R}$ [4], i.e., it is not a constant parameter defined by the receiver circuit specification. By also considering min./max. thresholds for rectenna activation and storage saturation, non-linear models have been formulated [14]. However, since we consider $P_{R}$ as a fixed value that has 
to be received by each $\mathrm{w}-\mathrm{p} D$ to sustain their operations, we proceed with the linear equation of $\eta$ by setting it as a constant and thus estimate the max. distance that the RF source can reach, at which the $\mathrm{w}-\mathrm{p} D$ s receive the min. $P_{R^{e f f}}$ required.

Since RF sources are presumed to radiate in nonoverlapping circular areas of $\pi R_{R F}^{2}$, the number of RF sources $k$ required to cover an event area of size $\Delta$ is simply calculated by $k=\Delta / \pi R_{R F}^{2}$, where the $\mathrm{w}-\mathrm{p} D \mathrm{~s}$ are accepted to be placed in $R_{R F}$ vicinity of a source. In other terms, $\Delta$ is covered without leaving any $\mathrm{w}-\mathrm{p} D$ in holes, i.e., dotted zones in Fig. 1b, occurred due to circular coverages of the sources.

By using $R_{R F}$ from (1) in consideration of (2), $k$, with respect to $\Delta$, can be reformulated as

$$
k=\Delta / \pi\left(\frac{\eta P_{T} G_{T} G_{R} c^{2}}{16 \pi^{2} f^{2} P_{R^{e f f}}}\right)^{2 / \delta} .
$$

Note that, all RF sources are assumed to have the same antenna characteristics with omni-directional propagation.

In conclusion, (3) can be used to designate relevant design guidelines enabling effective power coverage and communication connectivity in the envisioned network architecture.

\section{NUMERICAL ANALYSIS}

This section investigates the maximum achievable coverage with minimum $k$, depending on RF source and $\mathrm{w}-\mathrm{p} D$ characteristics besides medium parameters, in regard to limiting factors, i.e., usable energy budget and the FCC regulations.

\section{A. RF source and $w-p D$ Characteristics}

As disclosed earlier, an RF source first harvests energy $E_{H}$ by exploiting the E-field, then transmits a great portion of it ( $E_{H}^{\prime}$ ) as $P_{T}$ to w-p $D$ s for a duration of $T_{T}$. In other words, $E_{H}^{\prime}=P_{T} \times T_{T}$, where the w-p $D$ s receive $P_{R}$ of this $P_{T}$ and use $P_{R^{e f f}}$ of it after rectification. Thus, the energy that the w-p $D$ s can effectively utilize $E_{R^{e f f}}$ is equal to $P_{R^{e f f}} \times T_{T}$. Although it seems $E_{R^{e f f}}$ is restricted only by $E_{H}^{\prime}$, there is another limit set by the FCC [15], which implies that the maximum power conveyed in indoor cannot exceed $4 \mathrm{~W}$ (or $36 \mathrm{dBm}$ ) of effective isotropic radiated power (EIRP) due to potential hazards for human body. EIRP can be simply defined as $P_{T}[\mathrm{dBm}]+G_{T}[\mathrm{dBi}]$ in terms of $d B m$ or $P_{T}[\mathrm{~W}] \times G_{T}$ in watts. Thus, $P_{T}$ and $G_{T}$ can be only altered as long as their product obeys the FCC rule, i.e., $P_{T} \times G_{T}=4 \mathrm{~W}$ EIRP, where $P_{T}$ is also limited by $E_{H}^{\prime}$, so the $E_{R^{e f f}}$.

If $E_{R^{e f f}}$ is set to $E_{R}^{\text {min }}$, i.e., the minimum energy required to properly operate sensing, processing and communication peripherals of an ultra-low-power transceiver [9] that the w$\mathrm{p} D$ has, then the $R_{R F}$ in (1) will give the maximum distance $R_{R F}^{\max }$ that the RF source can possibly reach. Considering this, we first analyze how the antenna gain of the source affects $R_{R F}^{\max }$ at which a w-p $D$ can collect $4 \mathrm{~mJ}$ of energy $E_{R}^{\min }$, needed for nodal operations and during sleep as explained in Section II-C. Therefore, for an average conversion efficiency $\eta$ of $80 \%$ in regard to state-of-the-art rectannas [4], the w-p $D$ has to accumulate $5 \mathrm{~mJ}$ of energy $E_{R}$ before rectification.

At first glance, it is believed that changing $G_{T}$ will not alter the achievable coverage as the output power in EIRP is set to $4 \mathrm{~W}$. However, since $T_{T}$ is also varying with $P_{T}$ due to fixed $E_{H}^{\prime}, R_{R F}^{\max }$ is different for each $P_{T}-G_{T}$ combination when aiming at a specific $E_{R}$. To be more precise, if $G_{T}$ of $6 \mathrm{dBi}$ is used, $P_{T}$ will be $1 \mathrm{~W}$ as meeting the $4 \mathrm{~W}$ EIRP rule, which will result in $2.5 \mathrm{sec}$ of $T_{T}$ considering $2.5 \mathrm{~J}$ of $E_{H}^{\prime}$ budget. In that case, the required $P_{R}$ for $E_{R}$ of $5 \mathrm{~mJ}$ is $2 \mathrm{~mW}$, where $R_{R F}^{\max }$ is $2.32 \mathrm{~m}$. However, when the antenna is changed to another with $G_{T}$ of $9 \mathrm{dbi}, P_{T}$ reduces to $0.5 \mathrm{~W}$ while $T_{T}$ increases to $5 \mathrm{sec}$ as equating the required $P_{R}$ to $1 \mathrm{~mW}$, where $R_{R F}^{\max }$ reaches up to $3.25 \mathrm{~m}$. As depicted in Fig. 2, same behavior is observed also for $G_{T}$ of $12 \mathrm{dBi}$, i.e., $R_{R F}^{\max }=4.56 \mathrm{~m}$. Even though $R_{R F}^{\max }$ increases with higher $G_{T}$, increasing antenna size becomes a limiting factor as well as the duty cycle, which gets higher with the lengthened $T_{T}$. Since smart building services necessitate certain reporting frequency, the increased duty cycle may not be tolerated always. Thus, these issues need further elaboration for optimal designation of relevant design parameters as achieving the best performance depending on the application/network requirements.

The above analysis is based on (1), where the RF source with $f$ of $0.902 \mathrm{GHz}$ periodically feeds the surrounding w$\mathrm{p} D \mathrm{~s}$ that have $6 \mathrm{dBi}$ of $G_{R}$. Again from Fig. 2, when $f$ is increased from 0.902 to $2.45 \mathrm{GHz}, R_{R F}^{\max }$ decreases to 0.87 , 1.22 and $1.71 \mathrm{~m}$ for 6,9 and $12 \mathrm{dBi}$ of $G_{T}$, respectively. This is mainly due to signal fading, which increases with the carrier frequency of the source. In similar, for a specific $P_{T}$, any increment in the aperture between the RF source and the w$\mathrm{p} D$ imposes a decrement in $f$ to ensure the $\mathrm{w}-\mathrm{p} D$ collects the minimum energy required $E_{R}^{\min }$. This is shown in Fig. 3a by the straight red line depicting the level of $5 \mathrm{~mJ}$, where any $R_{R F^{-}} f$ pair falling below this line fails to sustain the nodal cycles of the $\mathrm{w}-\mathrm{p} D$. Considering this and the fact that majority of commercial wireless devices operate in $2.4-2.454 \mathrm{GHz}$ band, keeping $f$ in sub-GHz is essential as it will not only increase $R_{R F}^{\max }$ but also reduce the probability of interference.

As indicated by (1), increasing $P_{T}$ increases $P_{R}$ for a fixed $f$ and $R_{R F}$. However, as the length of $T_{T}$ is inversely proportional to $P_{T}$ due to the fixed $E_{H}^{\prime}$, aiming at a certain $E_{R}$ dictates $P_{R}$ to reach a minimum level as meeting the predefined requirements. This, i.e., the effect of having a limited energy budget/transmission duration, is shown in Fig. 3b, where the red line marked with $\star$ gives $P_{T}$ specific minimum $P_{R}$ threshold (for $R_{R F}$ of $2.5 \mathrm{~m}$, which is set by considering the average floor-to-ceiling height of the typical

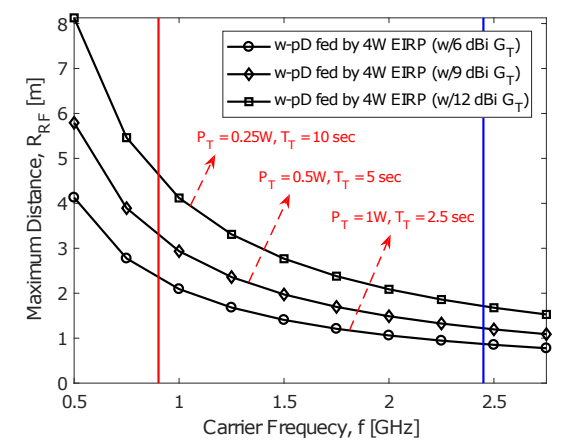

Figure 2: Maximum distance $R_{R F}^{\max }$ vs. carrier frequency $f$ of the RF source for varying $G_{T}$, where $\delta_{L o S}$ is 2.04 and $G_{R}$ is $6 \mathrm{dBi}$. 




(a)

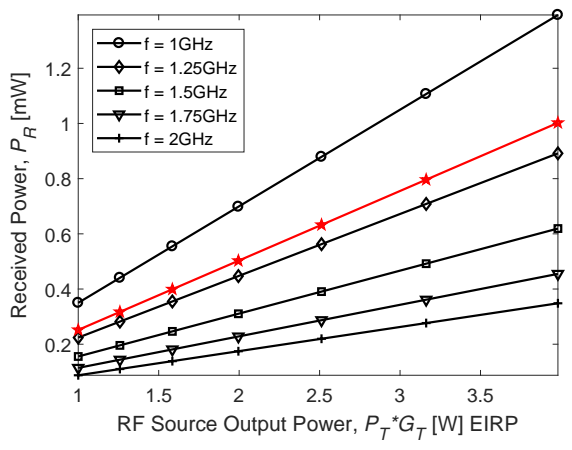

(b)



(c)

Figure 3: (a) $E_{R}$ vs. $R_{R F}$ for varying $f$ (at $4 \mathrm{~W}$ EIRP, LoS case); (b) $P_{R}$ vs. RF source output power for varying $f$ (at $R_{R F}$ of $2.5 \mathrm{~m}$, LoS case); (c) $k$ vs. $\Delta$ for varying $f$ (at $4 \mathrm{~W}$ EIRP, LoS and non-LoS cases where $\delta_{L o S}=2.04$ and $\delta_{N L o S}=2.94$ [13]).

office buildings). As seen, for higher $f$ (e.g., $f>1 \mathrm{GHz}$, specific to this scenario), the w-p $D$ fails to receive $P_{R}^{\min }$ determined by $P_{T}$ and $T_{T}$. For greater $R_{R F}$, black lines move downwards of the threshold line with a decreasing slope, which restricts the system further. For lesser $R_{R F}$, however, they go upwards with an increasing slope, where the operation is alleviated as a corollary of $P_{R} \propto 1 / R_{R F}^{2}$ relation.

Apart from $G_{T}, G_{R}$ could be also altered to increase $P_{R}$. However, since increasing gain refers to increasing antenna size, w-p $D$ s are equipped with antennae having $G_{R}$ of $6 \mathrm{dBi}$ to conserve small form factors. In similar, by considering the outcomes of the above-explained analysis besides off-the-shelf products, $G_{T}$ is selected as $9 \mathrm{dBi}$.

\section{B. Medium Parameters}

The previously disclosed factors, each of which increases $R_{R F}$, cause the number of $k$ ensuring successful coverage over $\Delta$ to decrease. As implied by (3), for a specific $P_{T}$ and a given $\Delta$, more RF sources are required at the higher frequencies of power transmission. In similar, increasing $\Delta$ signifies covering a larger field, which entails more sources to be deployed as $R_{R F}$ is restricted by $P_{T}$. However, if $P_{T}$, so the $R_{R F}$, is increased, RF sources become able to reach more $\mathrm{w}-\mathrm{p} D \mathrm{~s}$, where a smaller number of $k$ would be enough for effective coverage over the area of interest.

For the numerical analysis of these arguments, an RF source with $9 \mathrm{dBi}$ of antenna transmitting $0.5 \mathrm{~W}$ of constant power to the nearby w-p $D$ s with $G_{R}$ of $6 \mathrm{dbi}$ is assumed, in regard to limits set by the FCC and the employed EH technique as well as $E_{R}^{\min }$ required by the w-pDs. As seen in Fig. 3c, at the maximum permissible output power, i.e., $4 \mathrm{~W}$ EIRP, an event area of size $100 \mathrm{~m}^{2}$ can be covered by only $3 \mathrm{RF}$ sources $(k=3.012)$ for $f$ of $0.902 \mathrm{GHz}$. This number rises to 9 for $1.5 \mathrm{GHz}$ and to 15 for $2 \mathrm{GHz}$ in an $\operatorname{LoS}$ scenario. For the non-LoS case, 7, 13 and $19 \mathrm{RF}$ sources are required for $f$ of $1,1.5$ and $2 \mathrm{GHz}$, respectively. As revealed, $k$ is directly proportional to $f$ as signal fading becomes more disruptive at higher $f$, entailing an increment in $k$ for the same $\Delta$ due to the decreasing $R_{R F}^{\max }$. It should be noted that these results, generated by (3), refer to the worst-case scenario for duty cycling; however, well-planned models trading the reporting frequency with the number of RF sources may be achieved depending on application-specific requirements.

\section{CONCLUSION}

This study investigates the applicability of an energy-neutral IoT network, operation of which includes remote feeding of wireless devices with a limited source of power employing a domain-specific EH technique. Design guidelines for the practical implementation of such a concept are outlined by following inherent and obligatory restrictions. The findings attained based on empirical measurements disclose that conveying operational energy to the devices is theoretically achievable with a minimum number of sources possible. This opens up the potential of totally untethered and self-sufficient IoT services, which requires further efforts for its widespread utilization.

\section{REFERENCES}

[1] L. Atzori, A. Iera, and G. Morabito, "The Internet of Things: A survey," Computer Networks, vol. 54, no. 15, pp. 2787-2805, Oct. 2010.

[2] M.-L. Ku et al. "Advances in Energy Harvesting Communications: Past, Present, and Future Challenges," IEEE Communications Surveys \& Tutorials, vol. 18, no. 2, pp. 384-1412, Nov. 2016.

[3] O. B. Akan et al. "Internet of Hybrid Energy Harvesting Things," IEEE Internet of Things Journal, vol. 5, no. 2, pp. 736-746, Apr. 2018.

[4] X. Lu et al. "Wireless Networks with RF Energy Harvesting: A Contemporary Survey," IEEE Communications Surveys \& Tutorials, vol. 17. no. 2, pp. 757-789, second quarter 2015.

[5] E. B. Pehlivanoglu et al. "Harvesting-throughput trade-off for wirelesspowered smart grid IoT applications: An experimental study," IEEE International Conference on Communications (ICC), 2018, pp. 1-6.

[6] O. Cetinkaya and O. B. Akan, "Electric-field Energy Harvesting from Lighting Elements for Battery-less Internet of Things," IEEE Access, vol. 5, pp. 7423-7434, Apr. 2017.

[7] O. Cetinkaya et al., "Internet of Wireless-Powered Things: Analytical and Experimental Investigation of Energy Harvesting Wireless Autonomous Networks," submitted for publication.

[8] O. Cetinkaya and O. B. Akan, "Electric-field Energy Harvesting in Wireless Networks," IEEE Wireless Communications, vol. 24, no. 2, pp. 34-41, Apr. 2017.

[9] NXP Laboratories, "IEEE802.15.4 Wireless Microcontroller," JN5148001 datasheet, Oct. 2013.

[10] A. Bereketli and O. B. Akan, "Communication Coverage in Wireless Passive Sensor Networks," IEEE Communications Letters, vol. 13. no. 2, pp. 133-135, Feb. 2009.

[11] O. B. Akan et al., "Wireless Passive Sensor Networks," IEEE Communications Magazine, vol. 47, no. 8, pp. 92-99, Aug. 2009.

[12] C. A. Balanis, Antenna Theory: Analysis and Design, 2nd ed. John Wiley and Sons Inc., 1997.

[13] J. Lei et al., "Wireless Link SNR Mapping onto an Indoor Testbed," in Proc. of IEEE Tridentcom, 2005, pp. 130-135.

[14] E. Boshkovska et al., "Practical Non-linear Energy Harvesting Model and Resource Allocation for SWIPT Systems," IEEE Communications Letters, vol. 19, no. 12, pp. 2082-2085, Dec. 2015.

[15] FCC Codes of Regulation, Part 15. [Online]. Available: http://www. access.gpo.gov/nara/cfr/waisidx03/ 